\title{
PROPRIEDADES ELETROQUÍMICAS DE UM SOLO ELETROPOSITIVO INFLUENCIADAS PELA ADIÇÃO DE DIFERENTES FONTES DE MATÉRIA ORGÂNICA(1)
}

\author{
V. M. BENITES ${ }^{(2)}$ \& E. S. ME NDONÇA ${ }^{(3)}$
}

\begin{abstract}
RESUMO
Um Latossolo Vermelho-Amarelo com mineralogia predominantemente gibbsítica e com caráter eletropositi vo foi utilizado para estudos de propriedades eletroquímicas e mecani smos de dispersão, diante da adição de matéria orgânica. Foram utilizadas duas fontes orgânicas, caracterizadas como material não humificado (esterco) e humi ficado (ácido húmico extraído de turfa). Porções de solo foram incubadas com doses de 0; 7,5; 15,0; 22,5; e 30,0 $\mathrm{g} \mathrm{kg}^{-1}$ dos adubos orgânicos. Foram analisados argila dispersa em água, condutividade elétrica, pH em água e em $\mathrm{KCl}$, bem como determinados o ponto de efeito salino nulo (PESN) e o potencial elétrico superficial $\left(\Psi_{0}\right)$. Observou-se comportamento distinto entre os tratamentos com esterco e com ácidos húmicos em relação à dispersão de argi las. A adição de esterco provocou aumento da dispersão, o que pode estar relacionado com o aumento da condutividade elétrica pela presença de sais. Por outo lado, a adição de ácido húmico promoveu a floculação das argi las, em decorrência, provavelmente, da formação de complexos argilo-húmicos. 0 PESN decresceu linearmente com a adição dos adubos orgânicos, principal mente nos tratamentos com ácido húmico. A floculação não esteve obrigatoriamente associada a uma condição de baixo módulo de $\Psi_{\mathrm{o}}$. Nos tratamentos com doses mais elevadas de ácido húmico, foi observada carga negativa, apesar de não se observar dispersão de argila, provavelmente pelo fato de os ácidos húmicos apresentarem propriedades eletroquímicas distintas da matriz mineral, em função da sua condição de polieletrólito e da possibilidade de alteração na configuração da macromolécula.
\end{abstract}

Termos de indexação: adubação orgânica, argila di spersa em água, ponto de efeito salino nulo, Latossolos ácricos.

(1) Recebido para publicação em março de 1997 e aprovado em março de 1998.

(2) Doutorando do Curso de Solos e Nutrição de Plantas, Universidade Federal de Viçosa. Av. PH Rolfs, s/n. CEP 36571-000 Viçosa (MG). E-mail: vinicius@solos.ufv.br

(3) Professor Adjunto do Departamento de Solos, Universidade Federal de Viçosa. CEP 36571-000, Viçosa (MG). 


\title{
SUMMARY: ELECTROCHEMICAL PROPERTIES OF AN ELECTROPOSITIVE SOIL INFLUENCED BY THE ADDITION OF DIFFERENT ORGANIC MATTER SOURCES
}

\begin{abstract}
A gibbsitic Red-Yellow Oxisol with electropositivecarater was used to study the effect of organic matter on electrochemical properties and dispersi on mechanisms. Two organic sources were used, one characterized by non-humified compounds (cattle manure) and another by humified compounds (peat humic acids). The soil was incubated with $0 ; 7,5 ; 15,0 ; 22,5$ and $30,0 \mathrm{~g} \mathrm{~kg}^{-1}$ of organic manure Water dispersi bleclay, electrical conductivity, water and $\mathrm{KCl}$ $\mathrm{pH}$, and the zero point of saline effect (ZPSE) and superficial electric potential $\left(\Psi_{0}\right)$ were measured. Clay dispersion behavior was distinct when thesoil was treated with cattlemanure and humic acids. Soil dispersion increased with the addition of cattle manure, which may berel ated to theincrease of electrical conductivity dueto theamount of salts in themanure On the other hand, theaddition of humic acid induced clay flocculation, which was probably duetotheformation of clay-humi c complexes. TheZPSE linearly decreased with theaddition of organic manure especially with humic acids. The flocculation was not necessarily associated with thel ow modal condition of $\Psi_{\mathrm{o}}$. Negativechargewas observed in thetreatments with high levels of humic acids, but no clay dispersion was observed, probably due to the electrochemical properties of thehumic acids, as a result of its polyeletrolytic condition and the possibility of changing the macromol ecule configuration.
\end{abstract}

Index terms: organic manure, ZPSE, water dispersibleclay, acric Oxisol .

\section{NTRODUÇÃO}

O solo como um sistema disperso apresenta cargas el etroquímicas responsáveis pel os mecanismos dedispersão efloculação dos colóides e pela capacidade de troca catiônica e aniônica, entre outros processos. Essas cargas têm origem na fração mineral, sobretudo na fração argila, e na fração orgânica.

Em relação às cargas originadas nas argilas, observa-se uma diferenciação entre cargas variáveis e cargas permanentes. Por ação do intemperismo ocorrem o decréscimo das cargas permanentes e o aumento das cargas variáveis. A remoção da sílica e a presença deóxidos de FeeAl na fração argila levam à formação de cargas positivas denatureza variável (Morais et al., 1976; Gillman \& Uehara, 1980) e de natureza permanente (Tessens \& Zauyah, 1982). Em sol os tropicais bastante intemperizados, sobretudo em alguns Latossolos, pode-se observar o caráter ácrico, indicando, portanto, que, neste solo, o pH em $\mathrm{KCl}$ encontra-se acima do $\mathrm{pH}$ em água (Alleoni \& Camargo, 1994).

Para o estudo do comportamento el etroquímico dos solos, Schofield (1949) foi o primeiro a propor a determinação do ponto de carga zero (PCZ), como o $\mathrm{pH}$ em quea carga líquida de um sol o é igual a zero. Em valores de $\mathrm{pH}$ próximos ao PCZ, os colóides do solo apresentam baixo val or em módulo do potencial elétrico superficial, as forças de repulsão eletrostática são minimizadas, favorecendo a floculação (Gillman, 1974). Recentemente, Alleoni \& Camargo (1993) propuseram a utilização do termo ponto de efeito salino nulo (PESN), para identificar o valor de $\mathrm{pH}$ obtido no cruzamento de curvas de titulação potenciométrica, conforme Raij \& Peech (1972). Outros autores têm adotado esse termo por definirem o verdadeiro PCZ como o ponto no qual o balanço entre todas as cargas, inclusive as permanentes, seria zero, enquanto o PESN estaria relacionado somente com as cargas variáveis (Parker et al., 1979; Sposito, 1981). Dessa forma, com o pH no PCZ, a capacidade de troca catiônica se iguala à capacidade de troca aniônica na ausência de adsorção específica, enquanto no PESN ainda se manifestam as cargas permanentes (Sposito, 1984). Todavia, em solos que não apresentam argila de atividade alta, o PESN tem seu valor próximo ao PCZe, portanto, o PESN torna-seum bom parâmetro el etroquímico. Essa condição é observada em solos tropicais, sobretudo em L atossolos, compostos quase que exclusivamente por cargas variáveis (Uehara \& Gillman, 1980).

A adição de matéria orgânica a um sistema em equilíbrio irá promover alterações nas cargas em razão de fatores diretos e indiretos. A adsorção de ácidos orgânicos pel os colóides minerais irá causar um incremento das cargas negativas do sistema e, portanto, um abaixamento do PCZ (Oades, 1984). Por outro lado, a adição de matéria orgânica pode promover alterações no $\mathrm{pH}$ do solo, favorecendo a manifestação de cargas variáveis. Nos solos el etropositivos, esses efeitos se somam, levando-os à eletronegatividade, de forma que o ambiente el etropositivo dos horizontes subsuperficiais setorna el etronegativo próximo à superfície devido ao efeito 
da matéria orgânica (Coleman et al., 1989). A relação entre o potencial el etroquímico do meio ea dispersão de argila ainda é um fenômeno pouco estudado. A magnitude desse potencial está relacionada com a dispersão de argila na maioria dos casos, embora, em al gumas condições os mecanismos de dispersão não estejam diretamente relacionados com a condição el etrostática do meio, por causa de interações dos colóides inorgâni cos com substâncias húmicas (Oades, 1989; K retzchmar et al., 1993).

Nestetrabalho, procurou-seavaliar as diferenças qualitativas dos adubos orgânicos utilizados no manejo de solos tropicais, sobretudo sob o ponto de vista eletroquímico, e sua influência sobre a dispersão de argila em um Latossolo Vermel hoAmarelo com caráter ácrico.

\section{MATERIAL E MÉTODOS}

\section{Caracterização dos materiais utilizados}

Foi coletado, no município de Lassance (MG), o horizonte $B_{w}$ de Latossolo Vermelho-Amarelo distrófico argiloso (Quadro 1). Esse material apresentou caráter ácrico, baixo teor de matéria orgânica e mineralogia predominantemente gibbsítica, evidenciada por difração de raios $\mathrm{X}$.
Como fontes de matéria orgânica, foram utilizados esterco bovino fresco e ácido húmico comercial extraído deturfa, representando material não humificado e humificado, respectivamente (Quadro 2).

Os tratamentos foram distribuídos em um esquema fatorial $2 \times 5$, com três repetições. $O$ solo foi misturado com as duas formas orgânicas em cinco doses de $0 ; 7,5 ; 15,0 ; 22,5$ e $30,0 \mathrm{~g} \mathrm{~kg}^{-1}$ de adubo orgânico em base seca, correspondendo a adubação com 0, 15 , 30,45 e 60 t ha-1, respectivamente. Após um período de incubação de 90 dias, o material foi seco ao ar e submetido às análises.

\section{Análises químicas e físicas}

A argila dispersa em água (ADA) foi determinada segundo método da pipeta (E MBRAPA, 1979), sendo a dispersão mecânica promovida por agitador de baixo impacto durante 3 horas (J ucksch, 1987). A condutividade elétrica (CE) foi medida na suspensão de decantação de argila utilizada na avaliação de argila dispersa em água.

Foram determinados o $\mathrm{pH}$ em $\mathrm{H}_{2} \mathrm{O}$ e em solução $\mathrm{KCl} 1 \mathrm{~mol} \mathrm{~L}-1$, utilizando a proporção 1:2,5 v/v de solo:extrator. A partir desses valores, foi estimado o PCZ, conforme a equação proposta por Keng \& Uehara (1974):

$$
P C Z=2 \mathrm{pH} \mathrm{KCl}-\mathrm{pH} \mathrm{H}{ }_{2} \mathrm{O}
$$

Quadro 1. Caracteristicas(1) texturais e químicas do horizonte Bw de um L atossolo Vermelho-Amarelo de Lassance (MG)

\begin{tabular}{|c|c|c|c|c|c|c|c|c|c|c|c|c|}
\hline \multicolumn{3}{|c|}{ Análise textural } & \multicolumn{2}{|c|}{ pH } & \multirow{2}{*}{$\Delta \mathbf{p H}$} & \multirow{2}{*}{ C orgânico } & \multirow{2}{*}{$\mathrm{Ca}+\mathrm{Mg}$} & \multirow{2}{*}{ Al } & \multirow{2}{*}{$\mathbf{H}+\mathbf{A} \mathbf{I}$} & \multirow{2}{*}{$\mathbf{S}$} & \multirow{2}{*}{$\mathrm{CTC}_{\mathrm{pH}} 7$} & \multirow{2}{*}{$\mathbf{v}$} \\
\hline Areia & Silte & Argila & $\mathrm{H}_{2} \mathrm{O}$ & $\mathrm{KCl}$ & & & & & & & & \\
\hline 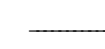 & $-\mathrm{g} \mathrm{kg}^{-1}$ & L & & & & $\mathrm{g} \mathrm{kg}^{-1}$ & 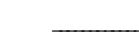 & 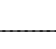 & $\mathrm{nol}_{\mathrm{c}} \mathrm{kg}^{-1}$ & 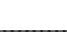 & L & $\%$ \\
\hline 330 & 260 & 410 & 5,1 & 5,6 & 0,5 & 5,8 & 4,0 & 0 & 12,0 & 5,0 & 17,0 & 29 \\
\hline
\end{tabular}

(1) Análises realizadas pelo laboratório de análises de rotina do Departamento de Solos da UFV, segundo EMBRAPA (1979).

Quadro 2. Caracterização química dos adubos orgânicos utilizados

\begin{tabular}{|c|c|c|c|c|c|c|c|}
\hline Adubo & Relação C/N $\mathbf{N}^{(\mathbf{1})}$ & $\mathrm{pH} \mathrm{H}_{2} \mathrm{O}$ & $\mathrm{MO}^{(2)}$ & $K^{(3)}$ & $\mathrm{Na}^{(3)}$ & $\mathrm{Ca}^{(3)}$ & $\mathbf{M g}^{(3)}$ \\
\hline & & & 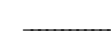 & 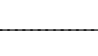 & $-\mathrm{g} \mathrm{kg}^{-1}$ & - & - \\
\hline Esterco & 40,4 & 7,6 & 798,9 & 13,2 & 17,5 & 2,8 & 2,3 \\
\hline Ácido Húmico & 19,9 & 6,8 & 355,7 & 8,5 & 8,5 & 2,7 & 4,0 \\
\hline
\end{tabular}


O ponto de efeito salino nulo (PESN) foi determinado, em duplicatas, pel o método de titulação potenciométrica descrito por Raij \& Peech (1972), utilizando-se como sal o $\mathrm{KCl}$, considerando suas propriedades de el etrólito simétrico (Sposito, 1981) e um tempo de equilíbrio de $24 \mathrm{~h}$, a fim de evitar o efeito paralelo da oxirredução da matéria orgânica (Siqueira, 1985). A adição de ácido ou base foi feita de forma que o cruzamento das curvas ocorresse próximo ao ponto médio, utilizando o PCZ estimado por Keng \& U ehara (1974) como referência.

Os dados de leitura de $\mathrm{pH}$ foram submetidos à análise pel o Programa Carga Zero (Fernandes Filho et al., 1995), o qual ajusta cada curva isoel etrolítica ao modelo hiperbólico e calcula a interseção entre estas (PESN). Em todos os tratamentos as curvas se ajustaram ao modelo com coeficiente de determinação $\left(R^{2}\right)$ superior a 0,94.

O potencial elétrico superficial $\left(\Psi_{0}\right)$ foi calculado, segundo Raij \& Peech (1972), utilizando-sea equação simplificada de Nernst:

$$
\Psi_{\mathrm{o}}=59,1(\mathrm{PESN}-\mathrm{pH}), \mathrm{em} \mathrm{mV}
$$

em que o PESN foi calculado tal como exposto, e o $\mathrm{pH}$ utilizado foi o $\mathrm{pH}$ em água.

Os dados foram anal isados estatisticamente pelo sistema deanál ises estatísticas egenéticas (SAEG), desenvolvido pela Universidade Federal de Viçosa. Foram testadas as correl ações entre o PCZ estimado e o PESN, e entre o $\Delta \mathrm{pH}$ e o $\Psi_{0}$. Por análise de regressão, foram determinadas as equações de regressão das variáveis estudadas em função das doses de adubo orgânico.

\section{RESULTADOS E DISCUSSÃO}

A adição dos adubos orgânicos ao solo provocou aumento linear da condutividade elétrica em decorrência da disponibilidade de quantidades apreciáveis desais. Esse efeito foi mais pronunciado nos tratamentos com esterco (Figura 1). A aplicação de 60 t ha-1 $^{-1}$ de esterco corresponde à adição de $1.050 \mathrm{~kg}$ de $\mathrm{Na}+$ e de $792 \mathrm{~kg}$ de $\mathrm{K}+$, segundo a caracterização do material (Quadro 2). Pratt (1979) observou o aumento da condutividade el étrica em solos adubados com doses el evadas de esterco, sobretudo pela presença de sais de sódio e potássio, atingindo níveis prejudiciais ao perfeito desenvolvimento das culturas.

O ponto de efeito salino nulo (PESN) decresceu linearmentecoma incorporação de matéria orgânica ao solo (Figura 2), concordando com os dados de outros autores (Siqueira, 1985; Siqueira et al., 1990). O PESN do sol o sem tratamento foi de 5,7, refletindo a mineralogia oxídica, aproximando-se dos valores de PCZ definidos para goethita e gibbsita de horizontes B latossólicos, de 6,4 e5,1, respectivamente
(Netto, 1996). O decréscimo do PESN deve-se à presença de grupos reativos da matéria orgânica, principalmente de grupos carboxílicos, que apresentam baixo pK e, portanto, se encontram dissociados, manifestando carga negativa na faixa de $\mathrm{pH}$ comum para os solos (Schnitzer, 1986). A presença desses grupos faz com que sejam necessários valores de $\mathrm{pH}$ mais baixos, para que parte da carga negativa da matéria orgânica seja suprimida, e o balanço de cargas atinja a nulidade, determinando o ponto de carga zero. Além desse efeito, parte das cargas positivas da matriz mineral pode ser anulada pela adsorção específica deânions orgâni cos, causando o abaixamento do PCZ (Oades, 1989).

Sabendo que são os grupos funcionais os responsáveis pelas propriedades el etroquímicas da matéria orgânica, torna-se necessária a consideração qual itativa dos adubos orgâni cos utilizados. Embora o teor de matéria orgânica seja maior no esterco, esta se compõe principalmente de lignocelulose e subprodutos pouco alterados. Tais compostos caracterizam-se por apresentarem baixa densidade de grupos funcionais por unidade de carbono, uma vez quetais grupos são formados pel a transformação oxidativa da lignina, com a formação dos grupos fenólicos e carboxílicos (Stevenson, 1982). Por outro lado, os ácidos húmicos extraídos de turfa representam um material com elevado grau de humificação, com a presença de grupos funcionais, cujas características refletem-se no seu comportamento el etroquímico. Dessa forma, o decréscimo do PESN foi mais pronunciado nos tratamentos com ácido húmico (Figura 2).

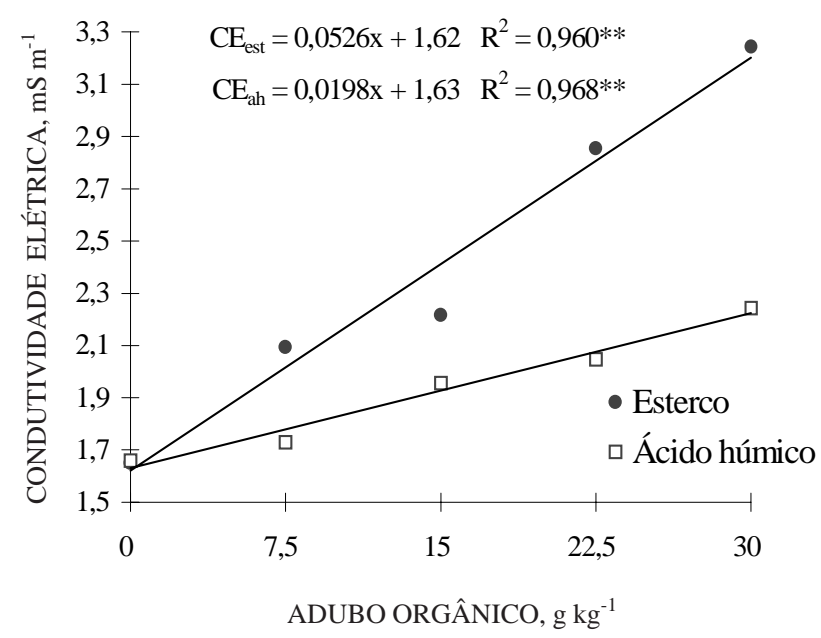

Figura 1. Condutividade elétrica (CE ) da suspensão de argila de um Latossolo Vermelho-Amarelo em função das doses de adubo orgânico recebidas. ${ }^{* *}$ significativo a $1 \%$ de probabilidade. 


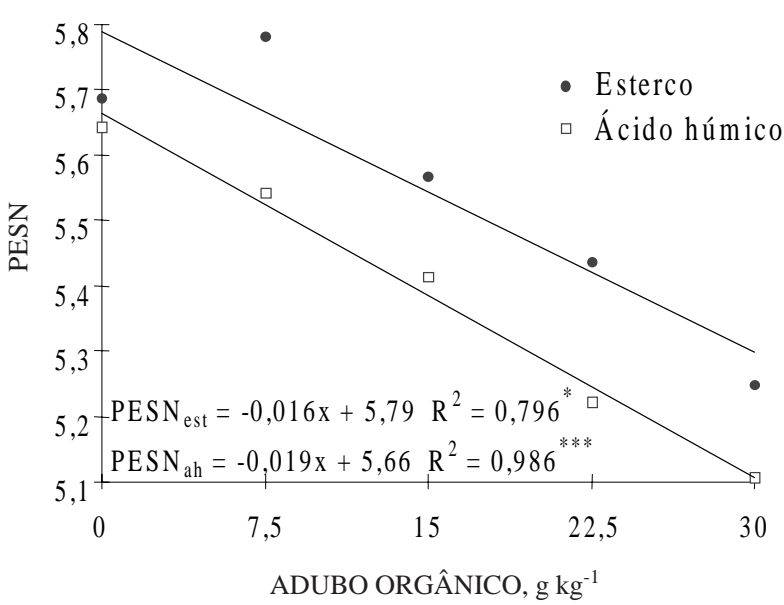

Figura 2. Ponto de efeito salino nulo (PESN) de um Latossolo Vermelho-Amarelo em função das doses de adubo orgânico recebidas. *, *** significativo a 5 e $0,1 \%$, respectivamente.

As correlações entre o PCZ estimado (eq.1) e o PESN foram significativas nos tratamentos com ácido húmico, tendo o PCZ estimado se correlacionado com o PESN, segundo equaçãolinear com inclinação igual a 1,00. Nos tratamentos com esterco, esta correlação não foi significativa, e os val ores de PCZ cal culados parecem ter subestimado o PESN nas doses mais elevadas (Figura 3).

Pela equação simplificada de Nernst (eq.2), pôdese verificar que o $\Delta \mathrm{pH}$ foi diretamente proporcional ao potencial elétrico superficial $\left(\Psi_{0}\right)$. Segundo Uehara \& Gillman (1980), o sinal ea magnitudedo $\Delta \mathrm{pH}$ estão relacionados com osinal e magnitude do $\Psi_{0}$. De fato, correlação significativa foi observada entre esses valores, tendo sido essa correlação nos tratamentos com ácido húmico $(r=0,9990,1 \%)$ mais significativa que a dos tratamentos com esterco $(r=0,9801 \%)$.

Os valores de $\Delta \mathrm{pH}$ e do potencial elétrico superficial decresceram com o aumento das doses de ambos os adubos orgânicos (Figura 4). Esse comportamento foi mais expressivo nos tratamentos com esterco, em que tanto o abaixamento do PESN quanto o aumento do $\mathrm{pH}$ com o aumento das doses contribuíram para o incremento de cargas negativas no meio. O solo, inicialmente el etropositivo, tornou-se eletronegativo com a adição de matéria orgânica. Estudando Latossolos ácricos, Alleoni \& Camargo (1994) encontraram valores negativos para o $\Delta \mathrm{pH}$ nos horizontes superficiais e valores positivos em subsuperficie. Coleman et al. (1989) demonstramque, em um perfil latossólico com mineralogia predominantemente oxídica, o horizonte A apresenta potencial elétrico negativo, provavelmente pela presença de matéria orgânica. $O$ potencial elétrico torna-se el etropositivo, à medida queseaprofunda no perfil, atingindo o que esses autores denominam de domínio oxídico. Nessa transição, em determinada profundidade, os val ores decarga líquida são próximos a zero, e os valores de argila dispersa são baixos.

O efeito deaumento de cargas el étricas negativas conferido pela matéria orgânica pôdeser evidenciado pela adição de esterco ao solo originalmente

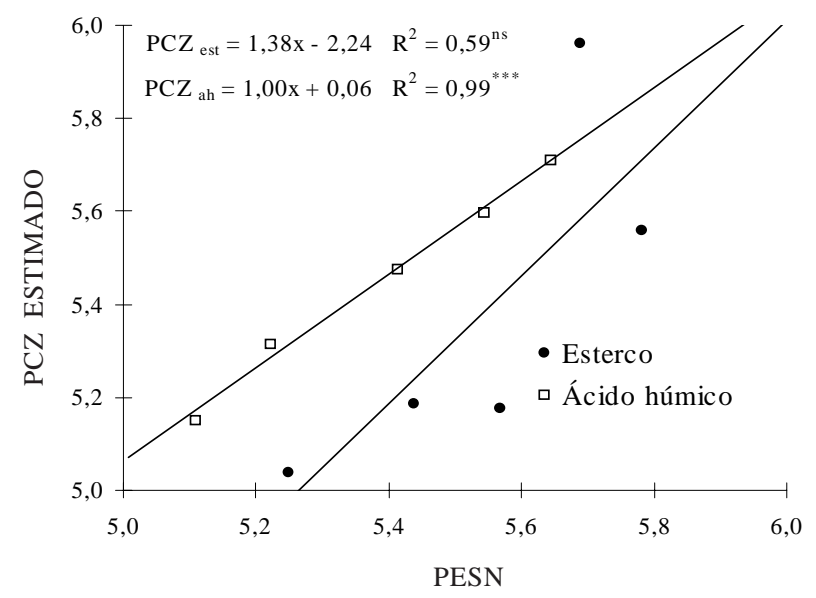

Figura 3. Ponto de carga zero estimado, nos tratamentos com esterco ( $\left.\mathrm{PCZ}_{\text {est }}\right)$ e com ácido húmico $\left(P C Z_{a h}\right)$, em função do ponto de efeito salino nulo (PESN). ns, *** não-significativo e significativo a $0,1 \%$, respectivamente.

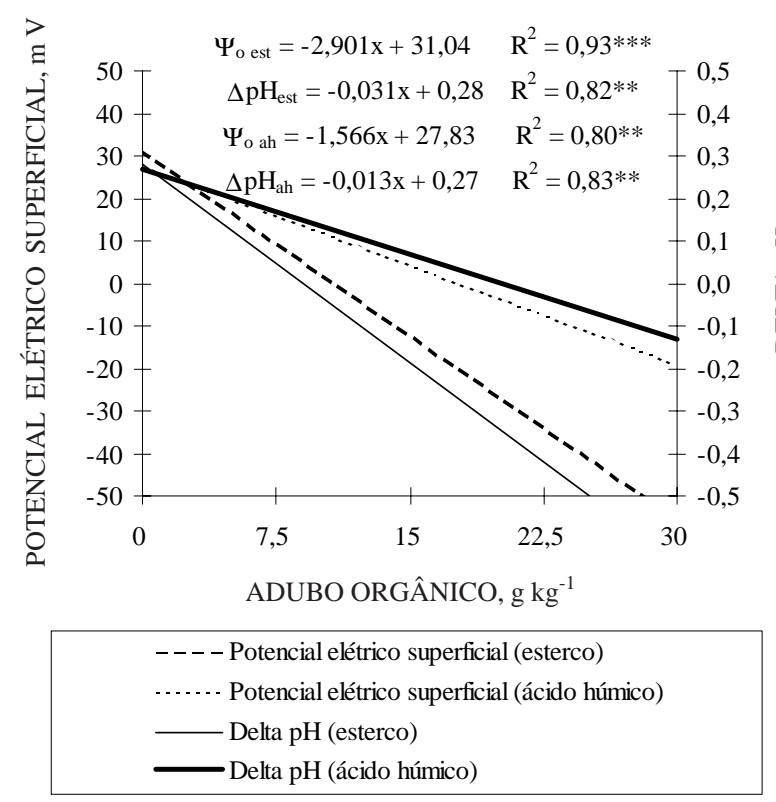

Figura 4. Potencial elétrico superficial ( $\Psi_{\text {o est }}$ e $\left.\Psi_{\text {oah }}\right)$ e Delta $\mathrm{pH}\left(\Delta \mathrm{pH}_{\text {est }} \mathrm{e} \Delta \mathrm{pH}_{\mathrm{ah}}\right)$ em função das doses de esterco e ácido húmico, respectivamente. **, *** significativo a 1 e $0,1 \%$, respectivamente. 
el etropositivo. Nesse caso, a variação em módulo do potencial elétrico superficial acompanhou o comportamento da dispersão de argila em água (Figura 5a). Enquanto os valores el etropositivos do solo sem tratamento ocasionaram a dispersão de argila, a adição das doses de 7,5 e $15,0 \mathrm{~g} \mathrm{~kg}^{-1}$ favoreceu a floculação. Com a adição de doses mais el evadas, passou-se a observar efeito dispersivo provocado pelo balanço negativo de cargas. 0 aumento da condutividade elétrica parece ter influenciado a maior dispersão nas doses extremas. A presença de sais em altas concentrações favorece a expansão da dupla camada difusa dos colóides, tornando-os mais susceptíveis à dispersão.

Nos tratamentos com ácido húmico, observou-se a tendência de aumento das cargas negativas, embora pouco expressiva, com o aumento das doses. Contudo, a presença das cargas negativas não se traduziu em fenômenos de repulsão entre as partículas. Ao contrário, o que se observou foi a floculação das partículas com o aumento das doses atingindo um patamar de quase $100 \%$ de floculação nas maiores doses (F igura $5 b$ ). Nos ácidos húmicos, as cargas são formadas nos grupos funcionais que estão distribuídos ao longo de toda macromolécula.

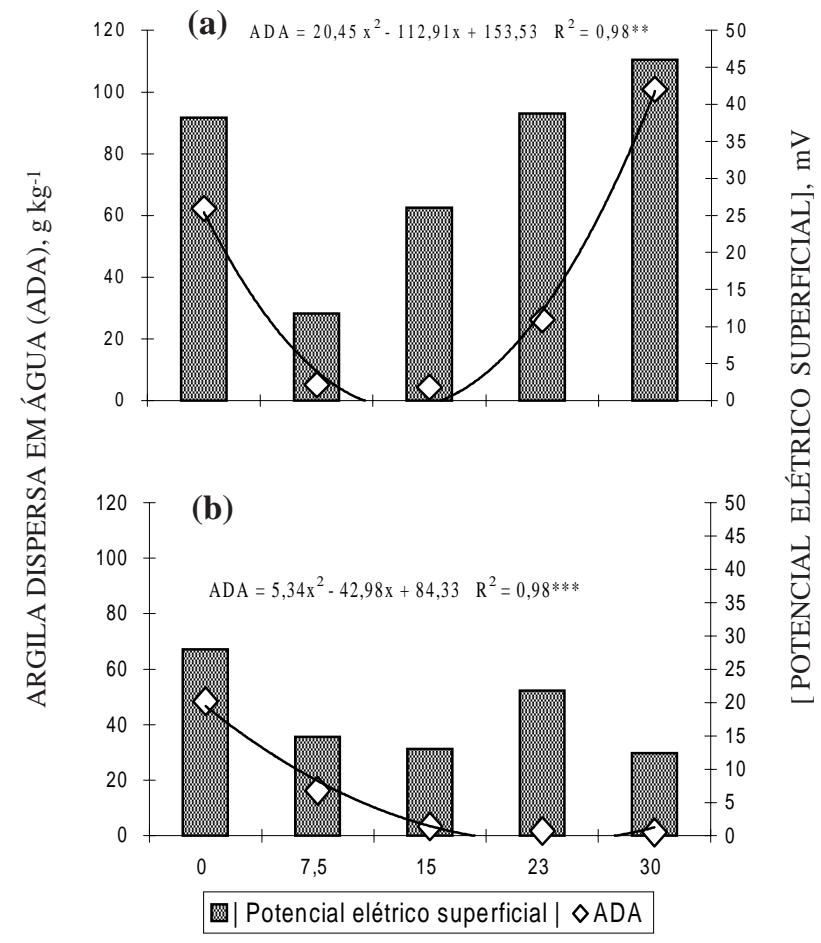

Figura 5. Argila dispersa em água (ADA) e módulo do potencial elétrico superficial de um Latossolo Vermelho-Amarelo em função das doses de: (a) esterco e (b) ácido húmico. **, *** significativo a 1 e 0,1\%, respectivamente.
As forças el etrostáticas que atuam entreessas cargas fazem com que a molécula sofra deformações, podendo apresentar-se na forma linear ou espiral izada de acordo com as condições de pH, força iônica e concentração de substâncias húmicas no meio (Ghosh \& Schnitzer, 1980). Essa propriedade é responsável pela preci pitação de complexos formados por ácidos húmi cos em pH inferior a 6,5 (Schnitzer, 1986), uma vez que, nessas condições, grande parte dos grupos funcionais não estão dissociados, e a molécula encontra-se na forma espiralizada.

Decorrentes dessas deformações, ocorrem fenômenos de natureza estérica, e parte das cargas do ácido húmico pode-se distanciar da superfície do colóideorgânico, reduzindo seu potencial de repulsão el etrostática, sem deixar de participar das reações de troca com os íons determinadores de potencial. Kretschmar et al. (1993) observaram a redução do efeito da matéria orgânica na estabilização de suspensões de argila em mei o com pH inferior a 6,0, sem que a mobilidade el etroforética da suspensão fosse alterada, atribuindo a este comportamento efeitos estéricos da matéria orgânica. Dessa forma, as argilas complexadas pelo ácido húmico preci pitam, sem que ocorra a neutralização de todas as cargas orgânicas. Além desses processos, as macromoléculas orgânicas podem promover a agregação de partículas pela ligação simultânea com duas ou mais superfícies, sem que haja a neutralização total das cargas. E mbora as forças de repulsão el etrostática continuem atuando, as forças de ligação organominerais podem-se sobrepor a estas, evitando a dispersão (Oades, 1984).

\section{CONCLUSÕES}

1. Do ponto de vista de manejo, a matéria orgânica pode apresentar efeito dispersivo ou agregante, de acordo com a quantidade e quali dade do adubo.

2. O uso de ácido húmico nas doses estudadas favoreceu as condições físicas de sol os el etropositivos, reduzindo a dispersão de argilas.

3. O valor de $\mathrm{PCZ}$ estimado a partir dos valores de $\mathrm{pH}$ em $\mathrm{H}_{2} \mathrm{O}$ e em $\mathrm{KCl}$ demonstrou ser bom avaliador do PESN, e o $\triangle \mathrm{pH}$, bom estimador do potencial elétrico superficial.

\section{LITE RATURA CITADA}

ALLEONI, L.R.F. \& CAMARGO, O.A . Ponto de efeito salino nulo de Latossolos ácricos. R. Bras. Ci. Solo, 18:175-180, 1994.

ALLEONI, L.R.F. \& CAMARGO, O.A. Ponto de efeito salino nulo: proposição de nomendatura. B. Inf. SBCS, 18:5-11, 1993.

COLEMAN, D.C.; OADES, J .M. \& UEHARA, G. Dynamics of soil organic matter in tropical ecossystems. Honolulu, University of Hawaii, 1989. 249p. 
EMPRESA BRASILEIRA DE PESQUISA AGROPECUÁRIA EMBRAPA. Serviço Nacional de Levantamento e Conservacão de Solos. Manual de métodos de análise de solo. Rio de J aneiro, 1979. 249p.

FERNANDES FILHO, E.I.; RUIZ, H.A.; PAIVA, A.Q. \& SOUZA, A.P. Carga Zero: Software para determinação matemática do ponto de carga zero. In: CONGRESSO BRASILEIRO DE CIÊNCIA DO SOLOS, 25., Viçosa, 1995. Resumos Expandidos. Viçosa, Sociedade Brasileira de Ciência do Solo, 1995. p.206-207.

GHOSH, K. \& SCHNITZER, M. Macromolecular structures of humic substances. Soil Sci., 129:266-276, 1980.

GILLMAN, G.P. \& UEHARA, G. Charge caracteristics of soils with variable and permanent charge minerals; 11. Experimental. Soil Sci. Soc. Am. J ., 44:252-255, 1980.

GI LLMAN, G.P. The influence of net charge on water dispersible clay and sorbed sul phate. Aust. J. Soil Res., 12:173-176, 1974.

J UCKSCH, I. Calagem e dispersão de argila em amostra de um L atossolo Vermel ho-Escuro. Viçosa, Universidade Federal de Viçosa, 1987. 37p. (Tese de Mestrado)

KENG, J.C.W. \& UEHARA, G. Chemistry, mineralogy and taxonomy of Oxisols and UItisols. Proc. Soil Crop Sci. Soc., 33:119-126, 1974.

KRETZCHMAR, R.; ROBARGE, W.P. \& WEED, S.B. Flocculation of kaolinitic soil clays: effect of humic substances and iron oxides. Soil Sci. Soc. Am. J ., 57:1277-1283, 1993.

MORAIS, F.I.; PAGE, A.L. \& LUND, L.J. The effect of salt concentration and nature of electrolytes on the charge caracteristics of Brazilian tropical soils. Soil Sci. Soc. Am. J ., 40:521-527, 1976.

NETTO, A.R. Influência da mineralogia da fração argila sobre propriedades físico-químicas de solos brasileiros. Viçosa, Universidade Federal de Viçosa, 1996. 144p. (Tese de Mestrado)

OADES, J .M. An introduction to organic matter in mineral soils. In: DIXON , J.B.E., WEED, S.B., eds. Minerals in soil environments. Madison, Soil Science Society of America, 1989. p. 89-160.
OADES, J.M. Soil organic matter and structural stability: mechanisms and implications for management. Plant Soil, 76:319-337, 1984.

PARKER, J .C.; ZELASNY, L.W.; SAMPATH, S. \& HARRIS, W.G. A critical evaluation of the extention of zero point of charge (ZPC) theory to soil systems. Soil Sci Soc. Am. J., 43:668673, 1979.

PRATT, P.. Management restrictions in soil application of manure. J. Anim. Sci., 48:134-143, 1979.

RAIJ, B. van \& PEECH, M. Eletrochemical properties of some Oxisols and Alfisols of the tropics. Soil Sci. Soc. Am. Proc., 36:587-598, 1972.

SCHNITZER, M. Binding of humic substances by the soil colloids. In: HUANG, P.M. \& SCHNITZER, M., eds. Interactions of soil minerals with natural organics and microbes. Madison, Soil Science Society of America, 1986. p.77-102

SCHOFIELD, R.K. Effect of pH on electric charges carried by clay particles. J. Soil Sci., 1:1-8, 1949.

SIQUEIRA, C. Eletroquímica de sol os tropicais de carga variável; efeito da matéria orgânica. I taguaí, Universidade Federal Fluminense do Rio de J aneiro, 1985 113p. (Tese de Doutorado).

SIQUEIRA, C.; LEAL, J.R.; VELLOSO, A.C.X. \& SANTOS, G.A. Eletroquímica de solos tropicais de carga variável: II. Quantificação do efeito da matéria orgânica sobre o ponto de carga zero. R. Bras. Ci. Solo, 14:13-17,1990.

SPOSITO, G. The operational definition of the zero point of charge in soils. Soil Sci Soc. Am. J ., 45:292-297, 1981.

SPOSITO, G. The surface chemistry of soils. Oxford, Oxford University Press, 1984. 277p.

STEVENSON, F.J . Humus chemistry: genesis, composition and reactions. New York, J ohn Wiley, 1982. 443p.

TESSENS, E. \& ZAUYAH, S. Positive permanent charge in oxisols. Soil Sci. Soc Am. J ., 46:1103-1106, 1982.

UEHARA, G \& GILLMAN, G.P. Charge caracteristics of soils with variable and permanent charge minerals: I Theory. Soil Sci. Soc. Am. J., 44:250-252, 1980. 